\title{
Parkin deficiency elevates hepatic ischemia/reperfusion injury accompanying decreased mitochondrial autophagy, increased apoptosis, impaired DNA damage repair and altered cell cycle distribution
}

\author{
XIAO-JIE NING ${ }^{1}$, XIONG YAN ${ }^{1}$, YAN-FENG WANG ${ }^{1}$, REN WANG $^{1}$, \\ XIAO-LI FAN ${ }^{1}$, ZI-BIAO ZHONG ${ }^{1}$ and QI-FA YE ${ }^{1,2}$

\begin{abstract}
${ }^{1}$ Hubei Key Laboratory of Medical Technology on Transplantation, Institute of Hepatobiliary Diseases, The Third Xiangya Hospital of Central South University, Changsha, Hunan 410013, P.R. China
\end{abstract} \\ Zhongnan Hospital of Wuhan University, Transplant Center of Wuhan University, Wuhan, Hubei 430071; \\ ${ }^{2}$ Research Center of National Health Ministry on Transplantation Medicine Engineering and Technology,
}

Received April 7, 2016; Accepted March 13, 2017

DOI: $10.3892 / \mathrm{mmr} .2018 .9606$

\begin{abstract}
Hepatic ischemia/reperfusion injury (HIRI) serves a causative role in postoperative hepatocyte death; however, the mechanisms underlying HIRI remain unclear. Mitochondrial autophagy, with apoptosis, cell cycle distribution and DNA damage repair, may be regarded as a regulatory factor post-HIRI. Parkin, a novel ubiquitin ligase, has been reported to increase mitochondrial autophagy and decrease apoptosis. However, the association between Parkin, mitochondrial autophagy and other regulatory factors in HIRI is unclear. In the present study, the effects of Parkin on HIRI were investigated, using hepatocytes and livers from male Sprague Dawley rats subjected to simulated in vivo HIRI. The results of the present study demonstrated that Parkin expression and mitochondrial autophagy were upregulated post-HIRI, leading to decreased hepatocyte death. Parkin knockdown suppresses the level of mitochondrial autophagy and promotes hepatocyte apoptosis by suppressing apoptosis regulator Bcl-2 function post-HIRI. In addition, Parkin deficiency alters cell cycle distribution and impairs DNA damage repair post-HIRI. In conclusion, Parkin facilitates mitochondrial autophagy and DNA damage repair, inhibits apoptosis, and modulates the cell cycle, leading to increased hepatocyte survival, demonstrating that Parkin may act as a protective regulatory factor post HIRI.
\end{abstract}

Correspondence to: Professor Qi-Fa Ye, Hubei Key Laboratory of Medical Technology on Transplantation, Institute of Hepatobiliary Diseases, Zhongnan Hospital of Wuhan University, Transplant Center of Wuhan University, 169 Donghu Road, Wuhan, Hubei 430071, P.R. China

E-mail: janejames@126.com

Key words: hepatic ischemia/reperfusion, Parkin, mitochondrial autophagy, apoptosis, DNA damage repair, cell cycle

\section{Introduction}

Hepatic ischemia/reperfusion injury (HIRI), a major cause of mortality post-liver surgery, occurs in a variety of circumstances when liver blood flow is interrupted, including during liver transplantation, liver resection and shock, and is compounded by multiple factors including ATP depletion, activation of neutrophils/macrophages, formation and release of cytokines and DNA damage (1). However, the specific mechanisms and mediators involved in postoperative HIRI remain largely unknown (1). Previous evidence has suggested that mitochondrial autophagy may act as a protective factor in canine hepatic injury (2). Protective effects have additionally been observed in porcine livers, and murine liver injury is more severe in autophagy-deficient rats compared with wild type counterparts, while inhibition of autophagy resulted in more severe injury of hepatocytes and endothelial cells (3). Using an adenoviral vector, an additional previous study demonstrated that overexpressed autophagy resulted in sustained protection of hepatocytes from hepatocyte injury (4). This previous evidence demonstrates that mitochondrial autophagy has a negative correlation with HIRI.

The specific regulatory factors of mitochondrial autophagy have not been completely elucidated (5). However, increasing numbers of studies have linked mitochondrial autophagy function to Parkin (6-8). In patients with Parkin mutations, the activity of the mitochondrial complex has been demonstrated to be reduced in leucocytes; rat Parkin knockout models exhibit reduced mitochondrial proteins and certain mitochondrial deficits, and an increased vulnerability towards autophagy function was observed in a Parkin deletion model (9). In addition, an association of Parkin with the stimulation of mitochondrial biogenesis has been observed in cells which overexpress Parkin, suggesting that Parkin serves an important and phylogenetically-conserved mitochondrial function (10). However, it remains unclear how Parkin mediates these effects on mitochondrial autophagy. 
In addition to mitochondrial autophagy, hepatocyte apoptosis, the biogenetic and metabolic process which serves a role in the initiation of cell death (11), contributes to HIRI as a key regulator, consistent with a previous study which demonstrated that short hairpin (sh)RNA-infected livers exhibited increased apoptosis compared with controls following HIRI (12). In a pig model, Parkin was able to protect hepatocytes from apoptosis and necrosis to improve survival during HIRI (13). Similar effects on apoptosis have been observed in the brain and intestinal tract (10). Previous experiments have identified that certain pro-apoptotic genes, including cellular tumor antigen p53, are upregulated in Parkin knockdown rats, and expression of the anti-apoptotic gene apoptosis regulator $\mathrm{Bcl}-2$ (Bcl-2) is altered. These previous data suggest that apoptosis is simultaneously regulated by Parkin (5). However, it remains unclear how Parkin deficiency triggers apoptosis. The regulatory effect of Parkin on mitochondrial autophagy and apoptosis may underlie the association between autophagy and apoptosis post-HIRI.

As important regulatory factors of cell death, DNA damage repair and cell cycle arrest are associated with liver injury (14). The initiation of DNA damage and unrepaired damage in the liver post-HIRI may be due to DNA double strand breaks (DSBs), which may directly lead to apoptosis, cause growth arrest and induce liver injury (15). In addition to the effects of DSB and G2/M cell cycle arrest, the upregulation of apoptotic genes contributes to the promotion of apoptosis and cell dysfunction during HIRI (16). The mechanisms involved in Parkin-mediated DNA damage repair and cell cycle arrest remain unclear.

The objective of the present study was to investigate the role of Parkin in HIRI and cell survival, particularly its influence on autophagy, apoptosis, DNA damage repair and cell cycle arrest. In an inducible under-expression system, the involvement of Parkin in the regulation of mitochondrial autophagy, apoptosis gene expression and the maintenance of the DSB repair protein was observed. It is hypothesized that these functions of Parkin contribute to the survival of normal hepatocytes post HIRI.

\section{Materials and methods}

Animals and ethics statement. Ninety adult male Sprague Dawley rats of 15-16 weeks of age (weighing 250-300 g) were obtained from the Animal Bio-Safety Level-III (ABSL-III) laboratory of the Wuhan University School of Medicine and were housed in accordance with the regulations of the National Institutes of Health (Bethesda, MA, USA). The animals were individually housed in stainless steel wire-bottomed cages and allowed access to standard chow diet and water, under a standard 12-h light/dark cycle. All surgical procedures were performed under sterile conditions. Care was taken to minimize suffering and pain. All of the animal experiments were approved by the Institutional Animal Care and Use Committee of Wuhan University (Wuhan, China).

Parkin knockdown vector construction. The shRNA was designed according to the Parkin sequence in the NCBI database (www.ncbi.nlm.nih.gov/). The fitted target sequence was selected in the coding region and exhibited no homology to any other gene, as observed using the basic local alignment search tool. The shRNA contained a unique 19-nucleotide double-stranded human Parkin sequence, which was presented as an inverted complementary repeat loop with a 9-nucleotide spacer and the concrete sequence GCCCCGATA TTTAAGCAAA and scramble sequence GACTCTCCGAAC GTGTCAC. The shRNA was cloned into plasmid pGenesil-1 (Genesil, Wuhan, China; www.genesil.com/)to generate the novel vector pshRNA-Parkin.

HIRI model and transfection. The HIRI model was constructed according to the method of Ohmori et al (17). A 5-cm midline incision was made on the abdominal wall of rats under brief light diethyl ether anesthesia $(20 \mathrm{mg} / \mathrm{l})$ according to the Ohmori et al (17). The portal pedicle, portal vein, and common hepatic artery were clamped for $20 \mathrm{~min}$ using a microclip. For the Parkin-knockdown experiments, the plasmids and the transfection agent Lipofectamine ${ }^{\circledR} 2000$ (Invitrogen; Thermo Fisher Scientific, Inc., Waltham, MA, USA) were mixed with Opti-MEM (Invitrogen; Thermo Fisher Scientific, Inc.) and incubated at room temperature for $5 \mathrm{~min}$, according to the manufacturer's protocol. The cultured SD rat hepatocytes were obtained according to the method described by Ohmori et al (17) were seeded following intraperitoneal injection with mixed shRNA plasmids $48 \mathrm{~h}$ prior to surgery. As a control, negative control plasmids were transfected into a subset of hepatocytes, and intraperitoneal injection was performed for the scramble group.

Reverse transcription-quantitative polymerase chain reaction $(R T-q P C R)$ analysis. All of the hepatocytes digested by pancreatin treatment $\left(25^{\circ} \mathrm{C}, 15 \mathrm{~min}\right)$ were harvested in order to extract mRNA using TRIZOL reagent (Invitrogen; Thermo Fisher Scientific, Inc.) according to the manufacturer's protocol. After a pre denaturation at $95^{\circ} \mathrm{C}$ for $30 \mathrm{sec}, \mathrm{qPCR}$ was performed using 35 cycles of denaturation at $95^{\circ} \mathrm{C}$ for $5 \mathrm{sec}$, annealing at $62^{\circ} \mathrm{C}$ for $20 \mathrm{sec}$ and elongation at $72^{\circ} \mathrm{C}$ for $30 \mathrm{sec}$. First strand cDNA synthesis was performed using a PrimeScript RT regent kit (cat. no. RR820A; Takara Bio, Inc., Otsu, Japan) and RT-qPCR analysis for Parkin was performed using the TaqMan kit (Applied Biosystems; Thermo Fisher Scientific, Inc.). The Parkin primer sequences were as follows: 5'-CCGAGTGACACTGATAGTGTTTGT-3' (forward) and 5'-ATCGTCTGCGGATTGGCTGTAGTT-3' (reverse); with the internal control gene GAPDH primer as follows: 5'-TCC TCTGACTTCAACAGCGACAC-3' (forward) and 5'-TCT CTCTTCCTCTTGTGCTCTTGG-3' (reverse). The relative expression was calculated using the $2{ }^{\Delta \Delta \mathrm{Cq}}$ method (18).

Immunoblotting. The hepatic tissues were washed twice with PBS and mixed with pancreatic treatment buffer. When the cells were homogenized, they were centrifuged at $13,400 \mathrm{x} g$ at $4^{\circ} \mathrm{C}$ for $5 \mathrm{~min}$. Total protein was extracted with Cell Lysis reagent (Pierce; Thermo Fisher Scientific, Inc.), quantified by the bicinchoninic acid method (Pierce; Thermo Fisher Scientific, Inc.), was incubated in loading buffer, and was boiled for $5 \mathrm{~min}$. Equal amounts of protein extracts (20 ul) were electrophoresed using SDS-PAGE on a $10 \%$ gel, and all the proteins were transferred to a polyvinylidene fluoride membrane and processed for immunoblotting. The membrane was blocked in 
$5 \%$ non-fat milk in TBS/Tween-20 for $2 \mathrm{~h}$ at $25^{\circ} \mathrm{C}$ and incubated with anti-Parkin antibody (cat. no. ab77924, 1:2,500; Abcam, Cambridge, UK), anti-microtubule associated protein 1 light chain 3 beta (LC3B) antibody (cat. no. 48397, 1:2,500; Abcam), anti BCl-2 antibody (cat. no. 59348, 1:2,500; Abcam), anti Ku-70 antibody (cat. no. 201963, 1:2,500; Abcam), anti Chk1 antibody (cat. no. 47574, 1:2,500; Abcam) or anti-beta actin antibody (cat. no. ab8226, 1:2,500; Abcam) overnight at $4^{\circ} \mathrm{C}$. Membranes were further incubated with horseradish peroxidase-conjugated (HRP) secondary antibody diluted (cat. no. ab6789, 1:5,000; Abcam) for $60 \mathrm{~min}$ at room temperature and bands were visualized using an enhanced chemiluminescence kit (Beyotime Institute of Biotechnology, Haimen, China). The autoradiographs were exposed onto X-Omat AR film (Kodak, Rochester, NY, USA). The density of bands in the films was quantified using Image J software (Version 2.1.4.7, National Institutes of Health).

Apoptosis and cell cycle assays. All of the hepatocytes digested by pancreatin treatment were harvested $48 \mathrm{~h}$ post-transfection, for apoptosis and cell cycle assays, and stained with $5 \mathrm{ml}$ annexin V-fluorescein isothiocyanate (FITC) and $5 \mathrm{ml}$ propidium iodide using the Annexin V-FITC Apoptosis Detection kit, with cell cycle distribution detected using the Cell Cycle Detection kit (Beyotime Institute of Biotechnology), according to the manufacturer's protocol. The percentage of apoptotic cells or cell cycle distribution was measured using flow cytometry with analyzing software (CytoDiff CXP 2.0; Beckman Coulter, Inc., Brea, CA, USA). All analyses were performed in triplicate.

Statistical analysis. All of the experiments were conducted in triplicate and data are expressed as the mean \pm standard deviation. Statistical analyses of data were performed using one-way analysis of variance with SPSS software (version 13.0; SPSS, Inc., Chicago, IL, USA) and GraphPad Prism software (version 6.0; GraphPad Software, Inc., La Jolla, CA, USA). P-values are based on two-sided hypothesis testing. $\mathrm{P}<0.05$ was considered to indicate a statistically significant difference.

\section{Results}

Parkin expression and mitochondrial autophagy are dramatically up-regulated following HIRI. The results of the present study demonstrated that the expression of Parkin mRNA was markedly upregulated following HIRI in a time dependent manner, with the most abundant expression $\sim 48 \mathrm{~h}$ post-HIRI (Fig. 1A). Similar to mRNA, Parkin protein expression was upregulated post-HIRI compared with the scramble control (Fig. 1B). The expression ratio of autophagy-associated proteins LC3-II/LC3-I, used as an indicator of mitochondrial autophagy, was upregulated in a time dependent manner post-HIRI (Fig. 1C), indicating an increased internal mitochondrial autophagy level post-HIRI.

Parkin knockdown suppresses the levels of autophagy during HIRI. The Parkin-knockdown experiment demonstrated that the quantity of Parkin protein in the Parkin knockdown group was markedly reduced by $>60 \%$ compared with the negative control group (Fig. 2A). The expression ratio of LC3-II/LC3-I
A

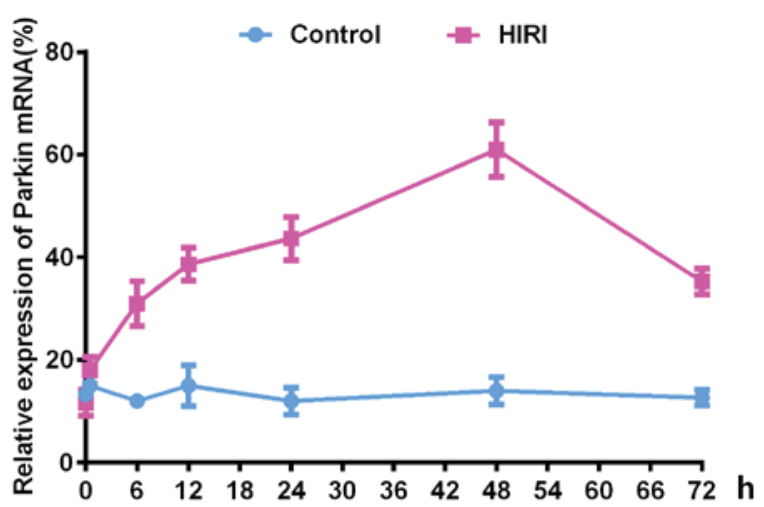

B

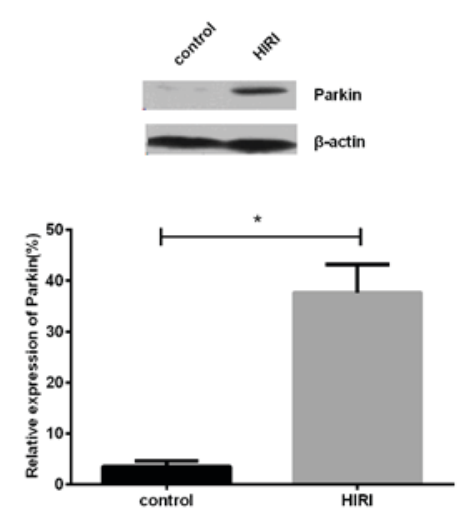

C

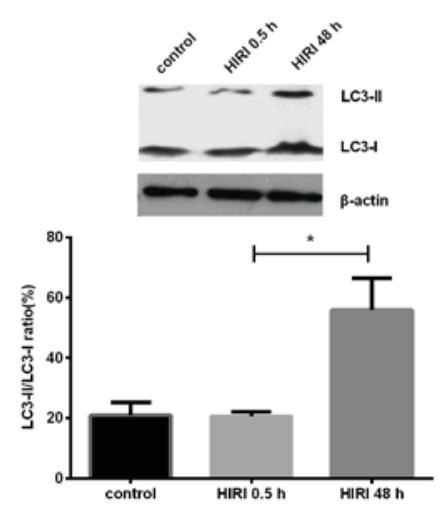

Figure 1. Parkin expression and mitochondrial autophagy are upregulated following HIRI. (A) Parkin mRNA is upregulated following HIRI in a time dependent manner, with maximum expression $\sim 48 \mathrm{~h}$ post-HIRI. (B) Western blot analysis demonstrates the protein expression of Parkin in rat hepatocytes treated with short hairpin RNA-Parkin and negative control. (C) Western blot analysis demonstrates the expression ratio of LC3-II/LC3-I in rat hepatocytes post-HIRI. The bar chart presents the semiquantitative analysis of Parkin expression. Data is presented as the mean \pm standard deviation. ${ }^{*} \mathrm{P}<0.05$. HIRI, hepatic ischemia/reperfusion injury; LC3, autophagy related protein LC3.

protein was reduced compared with the scramble control, in a time dependent manner (Fig. 2B). The results of the present study indicated that Parkin knockdown reversed the level of mitochondrial autophagy during HIRI.

Parkin knockdown promotes hepatocyte apoptosis by suppressing Bcl-2. In order to investigate the effect of Parkin on apoptosis during HIRI, Parkin protein was suppressed 
A
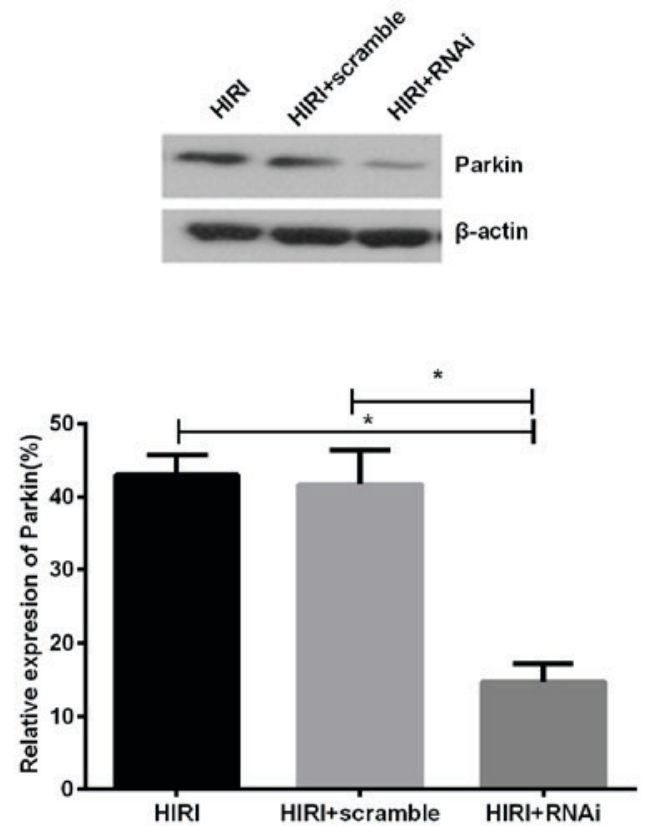

B
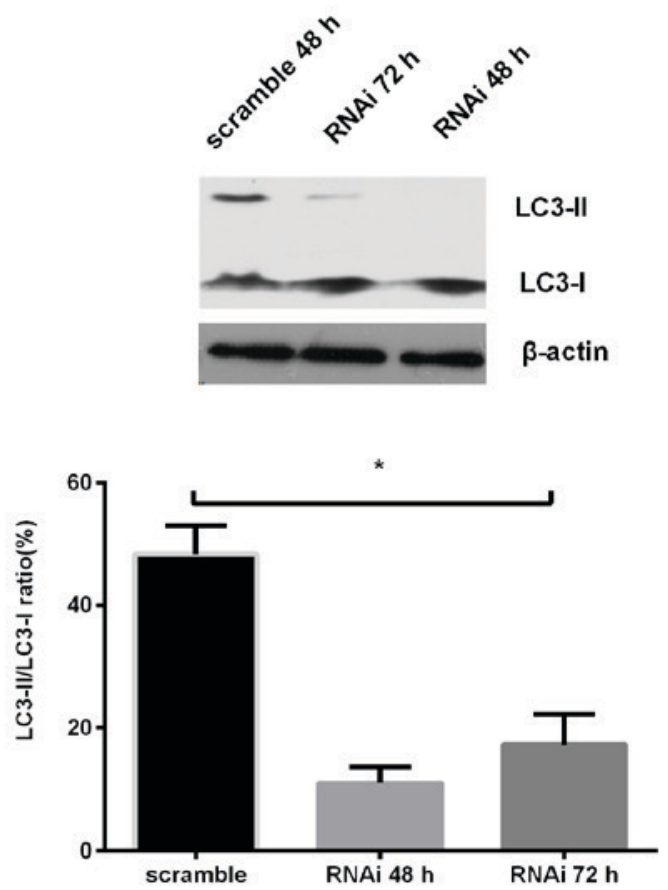

Figure 2. Parkin knockdown suppresses the level of mitochondrial autophagy following HIRI. (A) The level of Parkin protein in the Parkin knockdown group was decreased compared with the negative control group. (B) The relative expression of LC3-II/LC3-I was decreased compared with the scramble control in a time dependent manner. "P<0.05. HIRI; hepatic ischemia/reperfusion injury; LC3, autophagy related protein LC3; RNAi, RNA interference.

by shRNA-Parkin, the results of which indicated that the rate of apoptosis was markedly increased following Parkin knockdown (Fig. 3A). Bcl-2 expression was determined in rat hepatocytes transfected with shRNA-Parkin plasmid or scramble control using immunoblotting analysis, and the results demonstrated that $\mathrm{Bcl}-2$ expression was markedly downregulated in the knockdown group compared with the scramble group (Fig. 3B). The results of the present study
A
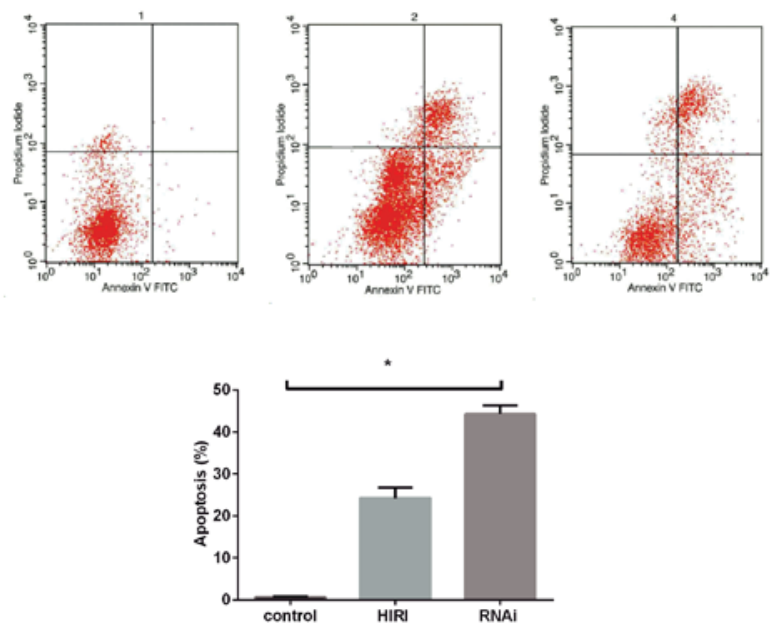

B
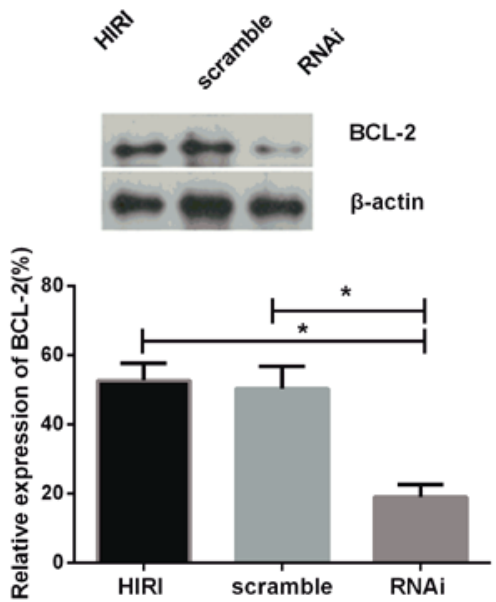

Figure 3. Parkin knockdown promotes hepatocyte apoptosis by suppressing Bcl-2. (A) The rate of apoptosis was increased following Parkin knockdown in HIRI. (B) The relative expression of Bcl-2 was decreased in the Parkin knockdown group compared with the scramble group. ${ }^{*} \mathrm{P}<0.05$. HIRI, hepatic ischemia/reperfusion injury; Bcl-2; apoptosis regulator Bcl-2; RNAi, RNA interference; FITC, fluorescein isothiocyanate.

suggested that Parkin deficiency may promote apoptosis by suppressing Bcl-2 expression in rat hepatocytes.

Parkin knockdown alters cell cycle distribution in HIRI. Following knockdown of Parkin expression, the proportion of cells in the G2/M phase decreased to 8.05, suggesting a decrease in $\mathrm{G} 2 / \mathrm{M}$ arrest (Fig. 4A). The expression of serine/threonine protein kinase chk1 (Chk1) post-HIRI was detected and the results demonstrated that, compared with the scramble control group, Chk1 expression was markedly reduced following Parkin knockdown (Fig. 4B). The results of the present study demonstrated that Parkin knockdown alters cell cycle distribution.

Parkin knockdown impairs process of DNA damage repair induced by HIRI. In order to investigate the effect of Parkin on the DNA damage repair response, expression of ATP-dependent DNA helicase II subunit 1 (Ku70), a typical DNA damage repair protein involved in the DSB repair mechanism, was determined in order to measure the level of DSB repair. The results of the present study demonstrate that the quantity of $\mathrm{Ku}-70$ in the Parkin knockdown group 
A
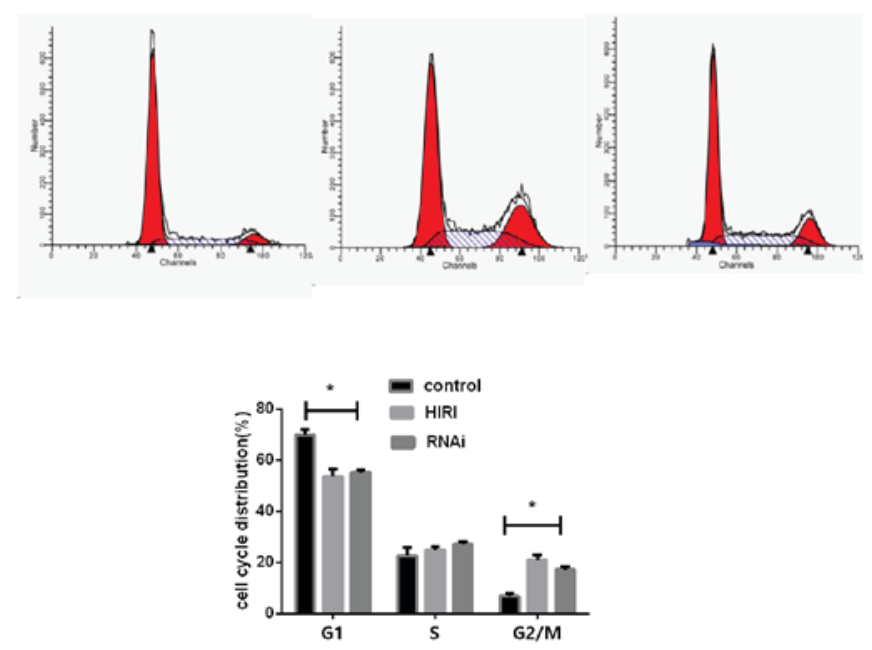

B

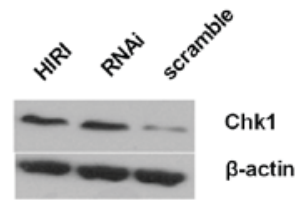

C
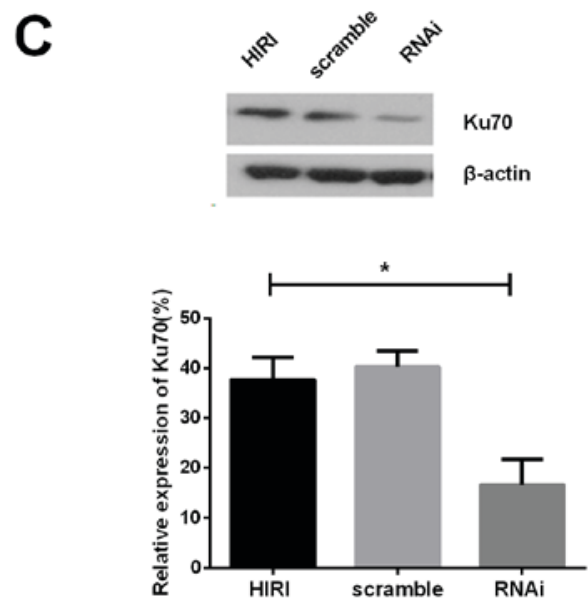

Figure 4. Parkin knockdown alters cell cycle distribution and impairs DNA damage repair in HIRI. (A) Following interference with Parkin expression, the proportion of cells in the G2/M phase decreased. (B) Expression of Chk1 was decreased following Parkin knockdown. (C) The expression of Ku-70 was decreased compared with the HIRI samples. "P<0.05. HIRI, hepatic ischemia/reperfusion injury; RNAi, RNA interference; Chk1, serine/threonine protein kinase chk1; Ku70, ATP-dependent DNA helicase II subunit 1.

was decreased compared with the HIRI samples (Fig. 4C). Therefore, Parkin deficiency may impair the DNA repair process in rat hepatocytes following HIRI.

\section{Discussion}

Parkin, exhibiting E3-ligase activity and mediating the covalent attachment of activated ubiquitin to target proteins, was initially hypothesized to function in a variety of cellular metabolic processes and numerous substitutes for Parkin have been identified, several of which are involved in HIRI (19). However, the definitive association of Parkin with proteasome-independent activities, which serve a role in mitochondrial autophagy, apoptosis, DNA damage response and HIRI, remains unclear (16).

Previous studies have investigated HIRI-induced liver damage with insufficient energy supply, which may be exacerbated by the degradation of mitochondrial autophagy (20). It is well established that mitochondrial autophagy and its derivatives potently and reversibly decrease mitochondrial transmembrane potentials and inhibit cell death in a concentration-dependent manner. The results of the present study demonstrate that mitochondrial autophagy may decrease cell death and liver injury post-HIRI; decreased levels of autophagy proteins and increased cell death following Parkin knockdown were observed post-HIRI. The results of the present study indicate that Parkin may function in the HIRI process as a protective factor, partly by promoting mitochondrial autophagy.

It has been previously demonstrated that there is an association between apoptosis and sensitivity to HIRI in mammals (4). Livers without injury have been demonstrated to exhibit decreased apoptosis compared with their counterparts, following HIRI and a direct effect of apoptosis on tissues is the suppression of energy production by mitochondria (21). At the gene expression level, certain studies (20) have reported that Bcl-2 secured correct rejoining of the DNA broken end to protect DNA integrity as DNA damage response post-HIRI, indicating that $\mathrm{Bcl}-2$-associated regulatory mechanisms serve an important role in HIRI; this may explain why Bcl-2 
downregulation results in increased sensitivity to HIRI. These previous studies suggest that the downregulation of anti-apoptotic genes and a subsequent increase in apoptosis may impair cell survival post HIRI (12). In the present study, Parkin inhibition induced Bcl-2 downregulation and an increase in apoptosis, which provides evidence that Parkin may be involved in Bcl-2 regulation and apoptosis control. Therefore, Parkin deficiency may cause a decreased efficiency in apoptosis suppression and increased cell death.

In addition to autophagy and apoptosis, certain studies have demonstrated that the G2/M cell cycle distribution may represent a novel mechanism of HIRI associated injury, wherein cell cycle arrest may provide sufficient time for cell damage to be repaired (16). Removal of Chk1, a specific marker of G2/M cell cycle arrest, or other cell checkpoint control proteins, may also contribute to injury (14). In the present study, following ischemia-reperfusion expression of Chk1 was measured; the results demonstrate that, compared with the control group, Chk1 expression was markedly reduced following Parkin knockdown, suggesting that Parkin is able to maintain the $\mathrm{G} 2 / \mathrm{M}$ arrest, in part, by regulating Chk1. However, the detailed mechanisms of Parkin function in cell cycle control remain unknown (22).

DNA damage and repair has been demonstrated to impact upon the outcome of HIRI (23). The repair of DSB, and the residual damage, appear to be important factors affecting HIRI. In the present study, DNA damage repair responses were observed in rat hepatocytes post-HIRI, following Parkin knockdown. The results of the present study demonstrated that there may be a negative association between DSB repair and Parkin, suggesting that Parkin may serve an important role in DSB recovery. However, the detailed mechanisms underlying the association between Parkin and DNA damage repair proteins remain unclear. The results of the present study provide a novel hypothesis that Parkin may function to protect cell survival by enhancing DSB repair and minimizing the residual DNA damage.

In conclusion, the results of the present study demonstrate physical evidence that Parkin deficiency may decrease the survival of hepatic cells, in addition to mitochondrial autophagy suppression, apoptosis promotion and DNA damage elevation, during the initiation and development of HIRI. The results of the present study demonstrate that Parkin serves a role in the induction of mitochondrial autophagy, the suppression of apoptosis, cell cycle distribution and DNA repair promotion during HIRI. The present data provide novel evidence that Parkin may act as a protective effector to protect hepatic cells from injury in the rat liver following HIRI.

\section{Acknowledgements}

The present study was supported by the Xinjiang Joint Funds of the National Natural Science Foundation of China (grant no. U1403222).

\section{References}

1. Teoh NC and Farrell GC: Hepatic ischemia reperfusion injury: Pathogenic mechanisms and basis for hepatoprotection. J Gastroenterol Hepatol 18: 891-902, 2003.

2. Kupiec-Weglinski JW and Busuttil RW: Ischemia and reperfusion injury in liver transplantation. Transplant Proc 37: 1653-1656, 2005.

3. Kao SY: DNA damage induces nuclear translocation of parkin. J Biomed Sci 16: 67, 2009.

4. Czaja MJ, Ding WX, Donohue TM Jr, Friedman SL, Kim JS, Komatsu M, Lemasters JJ, Lemoine A, Lin JD, Ou JH, et al: Functions of autophagy in normal and diseased liver. Autophagy 9: 1131-1158, 2013.

5. Thomas RL and Gustafsson AB: Mitochondrial autophagy-an essential quality control mechanism for myocardial homeostasis. Circ J 77: 2449-2454, 2013

6. Scarffe LA, Stevens DA, Dawson VL and Dawson TM: Parkin and PINK1: Much more than mitophagy. Trends Neurosci 37: 315-324, 2014.

7. Checler F, Goiran T and Alves da Costa C: Presenilins at the crossroad of a functional interplay between PARK2/PARKIN and PINK1 to control mitophagy: Implication for neurodegenerative diseases. Autophagy: 1-2, 2017.

8. Drapalo K and Jozwiak J: Parkin, PINK1 and DJ1 as possible modulators of mTOR pathway in ganglioglioma. Int $\mathbf{J}$ Neurosci 28: 102-104, 2017.

9. Miklya I, Göltl P, Hafenscher F and Pencz N: The role of parkin in Parkinson's disease. Neuropsychopharmacol Hung 16: 67-76, 2014.

10. Siriussawakul A, Zaky A and Lang JD: Role of nitric oxide in hepatic ischemia-reperfusion injury. World J Gastroenterol 16: 6079-6086, 2010.

11. Salminen A, Kaarniranta K, Kauppinen A, Ojala J, Haapasalo A, Soininen $\mathrm{H}$ and Hiltunen M: Impaired autophagy and APP processing in Alzheimer's disease: The potential role of Beclin 1 interactome. Prog Neurobiol 106-107: 33-54, 2013.

12. Sasaki H, Matsuno T, Nakagawa K and Tanaka N: Induction of apoptosis during the early phase of reperfusion after rat liver ischemia. Acta Med Okayama 51: 305-312, 1997.

13. Suzuki T, Yoshidome H, Kimura F, Shimizu H, Ohtsuka M, Takeuchi D, Kato A, Furukawa K, Yoshitomi H, Iida A, et al: Hepatocyte apoptosis is enhanced after ischemia/reperfusion in the steatotic liver. J Clin Biochem Nutr 48: 142-148, 2011.

14. Brenner C, Galluzzi L, Kepp O and Kroemer G: Decoding cell death signals in liver inflammation. J Hepatology 59: 583-594, 2013.

15. Zhang Y and Hunter T: Roles of Chk1 in cell biology and cancer therapy. Int J Cancer 134: 1013-1023, 2014.

16. Kao SY: Regulation of DNA repair by parkin. Biochem Biophys Res Commun 382: 321-325, 2009.

17. Ohmori M, Miyashita F, Uchida H, Kitoh Y, Tsuruoka S, Harada K, Sugimoto K, Fujimura A and Kobayashi E: Effect of erythromycin on ischemia-reperfusion injury of liver in rats. Transplant Proc 32: 811-814, 2000.

18. Livak KJ and Schmittgen TD: Analysis of relative gene expression data using real-time quantitative PCR and the 2(-Delta Delta C(T)) method. Methods 25: 402-408, 2001.

19. Du H, Yang W, Chen L, Shen B, Peng C, Li H, Ann DK, Yen Y and Qiu W: Emerging role of autophagy during ischemia-hypoxia and reperfusion in hepatocellular carcinoma. Int J Oncol 40: 2049-2057, 2012.

20. Hashmi SK, Baranov E, Gonzalez A, Olthoff K and Shaked A: Genomics of liver transplant injury and regeneration. Transplant Rev (Orlando) 29: 23-32, 2015.

21. Guicciardi ME, Malhi H, Mott JL and Gores GJ: Apoptosis and necrosis in the liver. Compr Physiol 3: 977-1010, 2013.

22. Dawson TM and Dawson VL: Parkin plays a role in sporadic Parkinson's disease. Neurodegener Dis 13: 69-71, 2014.

23. Cuervo AM and Wong E: Chaperone-mediated autophagy: Roles in disease and aging. Cell Res 24: 92-104, 2014. 